\title{
Dielectric relaxation behavior of nematic liquid crystal cell using $\beta$-cyclodextrin as an alignment layer
}

\author{
Marwa Sahraoui ${ }^{1, *}$, Asma Abderrahmen ${ }^{1}$, Rim Mlika $^{1}$, Hafedh Ben Ouada ${ }^{1}$ and \\ Abdelhafidh Gharbi ${ }^{2}$ \\ ${ }^{1}$ Laboratoire des Interfaces et Matériaux Avancés, Université Monastir, Faculté des Sciences de Monastir, \\ Département de Physique, Avenue de l'environnement 5000 Monastir, Tunisia \\ ${ }^{2}$ Laboratoire de la Matière molle, Faculté des Sciences de Tunis, Tunisia
}

\begin{abstract}
In the present investigation, we report the dielectric properties of a symmetric Nematic Liquid Crystal (NLC) cell using Beta Cyclodextrins ( $\beta-C D)$ as alignment layers. These layers were deposited onto Indium Tin Oxide (ITO) surface by thermal evaporation and then characterized using contact angle measurement. This revealed a hydrophilic character attributed to the presence of hydroxyl groups. Morphological study was carried out by Scanning Electronic Microscopy (SEM).

The dynamic impedance study of the Liquid Crystal (LC) cell in a wide frequency range from $1 \mathrm{mHz}$ to $13 \mathrm{MHz}$ was reported. It was found that the $\beta$-CD alignment layer had a blocking effect on the NLC cell at a high frequency range. We also report the relaxation mechanism of NLC cell which is modeled by an appropriate equivalent circuit in order to understand the electrical properties of the liquid crystal cell and to investigate the processes taking place at different interfaces.
\end{abstract}

Keywords: Liquid crystals; molecular dynamics; dielectric properties; interfaces.

\section{Introduction}

Liquid crystals (LCs) have been extensively investigated thanks to their increasing importance in technology, industry, science and medical applications. They have received considerable attention from many scientists over the last few decades ${ }^{1}$. These materials are used in electro-optical devices due to their anisotropic molecular order which can be controlled by external electric field ${ }^{2,3}$. It is well known that the dielectric anisotropy of the liquid crystals has an important impact on the image quality of Liquid Crystal Displays (LCDs) ${ }^{4,5}$. Moreover the high birefringence of liquid crystals is important for fast switching display ${ }^{6}$. For more advanced applications of LCs, it is necessary to understand the orientation of LC molecules and the effect of the surface on their alignment ${ }^{7}$. The anchoring properties of NLCs and their alignment on solid surfaces are the heart of most LC device applications $^{8,9,10}$.

Nowadays, there is a considerable interest in the use of macromolecules as components of LC devices. The particular features of these materials, which make them attractive for these applications, are their conformability, their low cost of fabrication, and the extreme ease of their deposition under thin film ${ }^{11}$.
Previous works discussed the possibility of using these macromolecules as potential materials to display devices. Peralta et al. have recently reported a reorientation of a liquid crystal induced by such a macrocyclic molecule like a calixarenes ${ }^{7}$. Cyclodextrins are a category of macrocyclic platform having a rigid natural cavity ${ }^{12,13}$, a good physical and chemical stability and compatibility with a variety field applications that can lead to low-cost devices. Up to now, they have been studied mostly for use in sensor devices and electronic device applications 11,14 . So, in this work, in order to develop a new liquid crystal device and to improve the dielectric properties and the alignment effect, Beta cyclodextrins are used for the first time as alignment layer. They are considered as an ideal candidate for this purpose due to their well defined structure ${ }^{13}$.

In this context, the NLC cell was fabricated using nematic liquid crystal (5CB) and Beta cyclodextrin $\quad(\beta-C D)$ as alignment layer. Before the manufacturing of NLC cell, the morphology of the films was characterized using Scanning Electron Microscopy (SEM). The relaxation dynamics and the dielectric properties of NLC cell were investigated. 


\section{Exprimental Section Materials}

The NLC used in this work is the polar liquid crystal (4- $n$-pentyl-4'-cyanobiphenyl) (commonly known as 5CB) which has a positive dielectric anisotropy. The $5 \mathrm{CB}$ is one of the best known liquid crystalline materials. It is in the nematic phase between $22.5^{\circ} \mathrm{C}$ and $35.5^{\circ} \mathrm{C}{ }^{15,16}$.

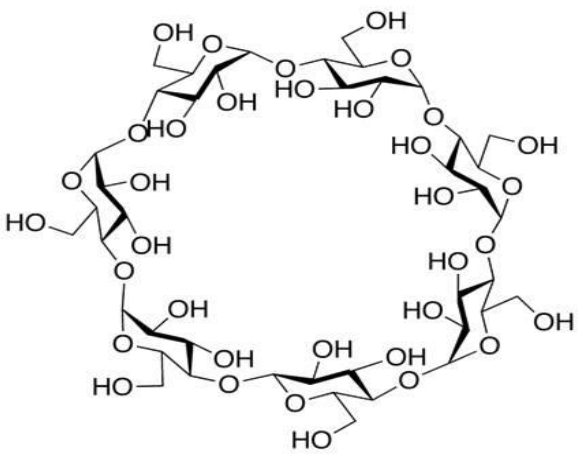

As an alignment layer, we chose the native Beta Cyclodextrin ( $\beta$-CD) $(99 \%$ Sigma Chemical Co, St Louis, Mo, USA). The native $\beta-C D$ is a bucketshaped oligosaccharide consisting of seven glucose units with a hydrophobic cavity and a hydrophilic exterior ${ }^{12,17}$. The chemical structure of the molecule is presented in Fig. 1.

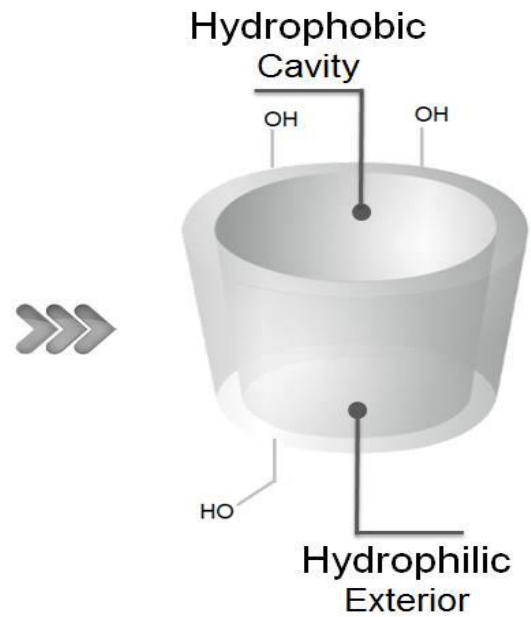

Figure 1. The molecular structure of $\beta$-Cyclodextrin.

Indium Tin Oxide (ITO) is usually used as an electrode material because of its transparency, high conductivity, good etchability, hardness and good adherence on many types of substrates. These characteristics allow its use in a wide range of optoelectronic devices, particularly LCDs ${ }^{9}$.

\section{NLC cell fabrication}

The NLC cell is prepared by two ITO-covered glass plates separated by Mylar sheets ${ }^{4,5}$. Firstly, before the deposition of the alignment layer, the native ITO-covered glass plates were pre-cleaned in acetone and methanol in ultrasonic bath for $20 \mathrm{~min}$ for each solvent ${ }^{9,18}$.

The substrates were finally dried under nitrogen flow to remove contaminants from the surface, improve adhesion of coating and prevent the humidity. The alignment layers of $\beta-C D$ were deposited onto ITO by thermal evaporation under secondary vacuum of $10^{-6}$ torr $^{13}$. During this process the temperature was maintained at about $200^{\circ} \mathrm{C}^{14}$ (evaporating temperature of the organic material), the evaporation rate and the deposited thickness were controlled by a piezoelectric quartz crystal microbalance. The measured deposited alignment layer thickness was about $300 \mathrm{~nm}$, measurements were carried out using dektak-3010 surface profilometer [13]. The liquid crystal 5CB was injected into the sample cells by capillary action ${ }^{4,5}$ at $25^{\circ}$, all samples were filled at room temperature. It is well known that $5 \mathrm{CB}$ liquid crystal has a nematic phase at room temperature ${ }^{15}$. The area and the thickness of the cell were $2.25 \mathrm{~cm}^{2}$ and $20 \mu \mathrm{m}$, respectively. The deposited alignment layer $\beta-C D$ favors a planar orientation of the LC molecules. This was checked through observation with a polarized microscope under a crossed polarizer-analyzer arrangement ${ }^{9}$.

\section{Measurements}

To characterize the $\beta$-CD layers, measurements of contact angle were performed with a model contact instrument (Dig drop) form GBX (Romans, France). The image of the liquid droplet behavior on the surface was taken with a digital camera and then analyzed ${ }^{13}$. Deionised water, formamide (Sigma Chemical Co, St Louis, Mo, USA) and diiodomethane (DIM) (Sigma Chemical Co, St Louis, Mo, USA) were used as the three liquid probes for surface free-energy calculations ${ }^{9}$. Van Oss theoretical model is an approach which has been widely used to measure wettability ${ }^{9,12}$, surface energy and its polar and dispersive components. A software based on Van Oss equation was used to calculate surface energies ${ }^{20}$.

A scanning electron microscopy (SEM), (JSM5400 Scanning Microscope) was used to study the surface topography of alignment layers.

The principle of Impedance Spectroscopy (IS) is to measure the electrical characteristics of a material as a function of the frequency of a small-amplitude AC signal. Changes in impedance characteristics with frequency provide information about conduction mechanisms, charge accumulation, 
dipole behavior and the dielectric relaxation of a material ${ }^{9,21}$.

In our study the dielectric measurements were carried out at ambient temperature and in a frequency range from $1 \mathrm{mHz}$ to $13 \mathrm{MHz}$. In the lower frequency range $(1 \mathrm{mHz}$ to $1 \mathrm{MHz})$, an impedance analyzer (Autolab PGSTAT) was used. The measurements of impedance in the frequency range from $5 \mathrm{~Hz}$ to $13 \mathrm{MHz}$ were taken using an HP 4192A impedance analyzer. For all the measurements, the amplitudes of the AC were $100 \mathrm{mV}^{9,13}$.

\section{Results and discussions}

\section{Contact angle measurement}

The wetting of the solid phase is always the first step in the preparation of optoelectronic devices. A wettability study was carried out before and after thin film deposition in order to check the non-

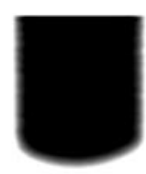

degradation of the deposited alignment layer after the deposition technique ${ }^{20}$. The results of liquid contact angle and surface energy of the samples are summarized in Table 1 . When the water contact angle on a surface is smaller than $90^{\circ}$, the surface is wetted by the water so the surface is hydrophilic ${ }^{20}$. We have found that the value of water contact angle value decreases from $\left(73^{\circ}\right)$ for the cleaned ITOcovered glass plates to $\left(46^{\circ}\right)$ for $\beta-\mathrm{CD}$ alignment layer (Fig. 2). This result proves that the surface became more hydrophilic. The reduction of the surface hydrophobicity can be attributed to the presence of the hydroxyl groups $(\mathrm{OH}$ : Hydrophilic functional) on the surface of the membrane ${ }^{22}$. On the other hand, we notice that the basic component $\gamma^{-}$ increases after the modification of ITO surface by the $\beta$-CD from $\left(8.3 \mathrm{~mJ} / \mathrm{m}^{2}\right)$ to $\left(35 \mathrm{~mJ} / \mathrm{m}^{2}\right)$, due to the presence of basic groups $(\mathrm{OH})$ (Table 1). This clearly proves that the alignment layers were well deposited $^{22}$.
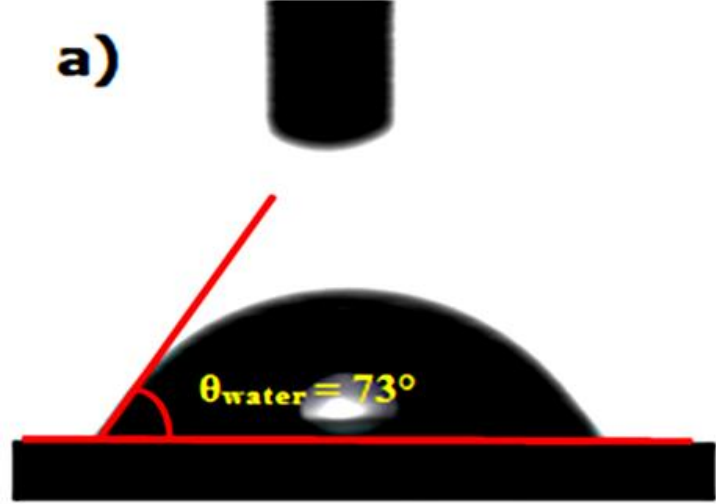

b)

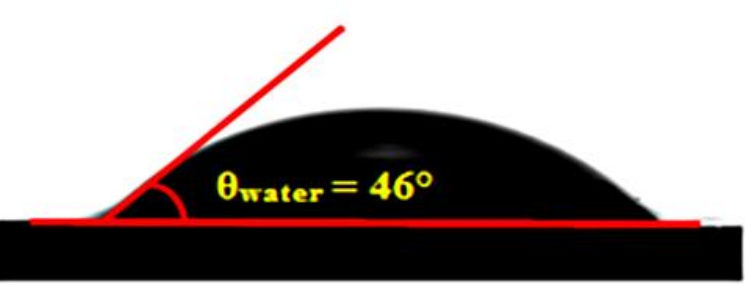

Figure 2. Water contact angle of a) Cleaned ITO electrode b) $\beta$-Cyclodextrin layer.

Table 1: Contact angle and surface energy data

\begin{tabular}{|c|c|c|c|c|c|c|c|c|}
\hline Sample & $\begin{array}{l}\boldsymbol{\Theta}^{\circ} \\
\text { water }\end{array}$ & $\begin{array}{l}\theta^{\circ} \\
\text { For }\end{array}$ & $\begin{array}{l}\boldsymbol{\theta}^{\circ} \\
\text { DIM }\end{array}$ & $\begin{array}{l}\gamma \mathrm{S} \\
\left(\mathrm{mJ} / \mathrm{m}^{2}\right)\end{array}$ & $\begin{array}{l}\gamma^{\mathrm{d}} \\
\left(\mathrm{mJ} / \mathrm{m}^{2}\right)\end{array}$ & $\begin{array}{l}\gamma^{\mathbf{p}} \\
\left(\mathrm{mJ} / \mathrm{m}^{2}\right)\end{array}$ & $\begin{array}{l}\gamma^{+} \\
\left(\mathrm{mJ} / \mathrm{m}^{2}\right)\end{array}$ & $\begin{array}{l}\gamma^{-} \\
\left(\mathrm{mJ} / \mathrm{m}^{2}\right)\end{array}$ \\
\hline Cleaned ITO & 73 & 50 & 35 & 44.6 & 42 & 2.6 & 0,2 & 8.3 \\
\hline$\beta$-CD native & 46.4 & 40.3 & 32.0 & 45.4 & 43.4 & 2.0 & 0.0 & 35.0 \\
\hline
\end{tabular}

$\mathrm{\Theta}^{\circ}$ water : water contact angle $\left({ }^{\circ}\right) ; \Theta^{\circ}$ For : formamide contact angle $\left(^{\circ}\right)$;

$\Theta^{\circ}$ DIM : diiodomethane contact angle $\left(^{\circ}\right) ; \gamma^{\mathrm{s}}=\gamma^{\mathrm{d}}+\gamma^{\mathrm{p}}:$ surface energy; $\gamma^{\mathrm{d}}:$ dispersive energy; $\gamma^{\mathrm{p}}=\left(2 \gamma^{+} \gamma^{-1 / 2}\right.$ : polar energy; $\gamma^{+}$: acid energy component; $\gamma^{-}$: basic energy component ${ }^{9,12}$.

\section{Surface Morphology Studies}

The realization of the desired orientation of the LC molecules depends on the alignment layers' morphology. So, before to manufacturing of the LC cell, the morphology of the films was characterized using scanning electron microscope. SEM images prove that the alignment layers were well deposited on the ITO (Fig. 3(a) and (b)). Additionally, the SEM image of the $\beta$-CD membrane (Fig. 3(b)) shows a compact structure and homogeneous surface 23. This is due to the high interaction between ITO substrate and the alignment film ${ }^{9}$. This morphology enormously affects the alignment of liquid crystal molecules ${ }^{1}$. 


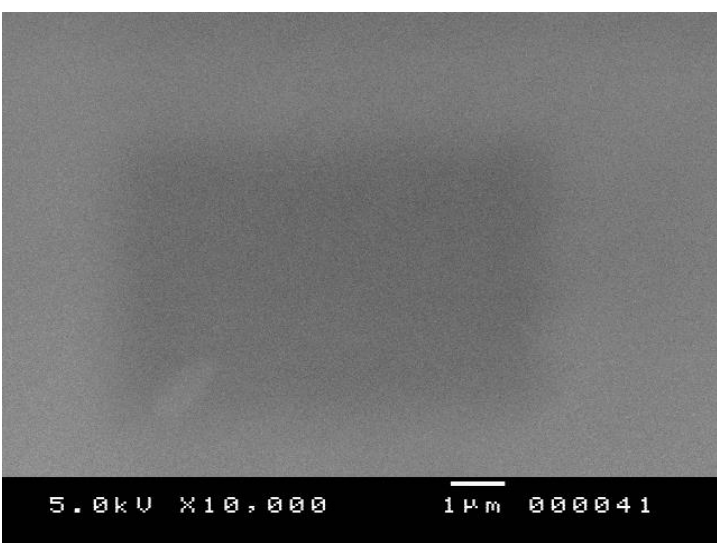

a)

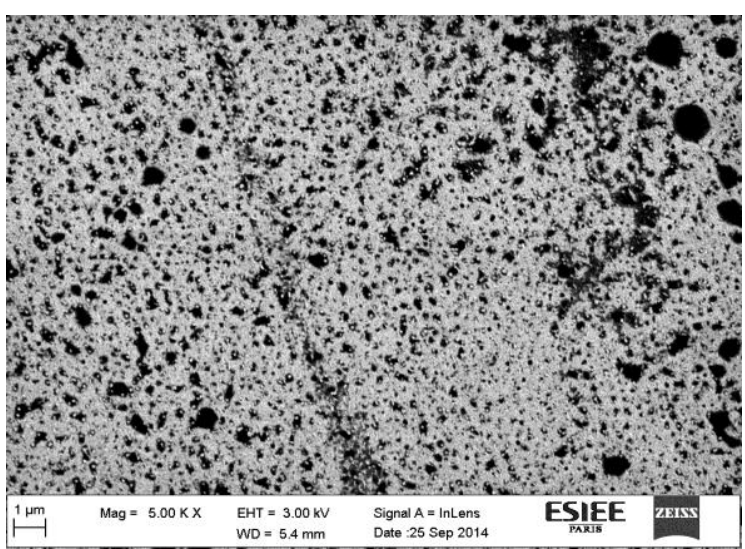

b)

Figure 3. SEM images of a) cleaned ITO electrode b) $\beta$-Cyclodextrin layers.

\section{Impedance spectroscopy}

Impedance Spectroscopy (IS) technique is an effective tool for the qualitative and quantitative characterization of the electrical processes occurring conducting NLC cells. Moreover, it can be used to study the interfacial properties of modified alignment layer. In the present work, the applied DC bias voltage was chosen higher than or equal to $2 \mathrm{~V}$ to avoid the diffusion effect observed at $0 \mathrm{~V}$ and $1 \mathrm{~V} \mathrm{DC}$ 9

\section{Conductance measurements}

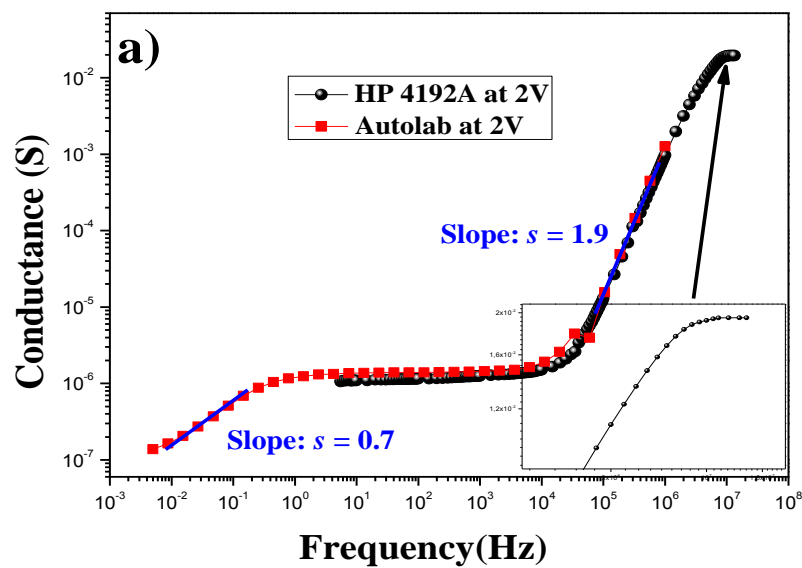

Fig. 4(a) shows the dependence of the conductance on frequency from $1 \mathrm{mHz}$ to $13 \mathrm{MHz}$ of the ITO $/ \beta-\mathrm{CD} / 5 \mathrm{CB} / \beta-\mathrm{CD} / \mathrm{ITO}$ cell at $2 \mathrm{~V}$. In such materials, the frequency dependent conductivity $G$ $(\omega)$ is expressed as the following equation ${ }^{24}$ :

$\mathrm{G}(\omega)=\mathrm{G}_{\mathrm{dc}}+\mathrm{G}_{\mathrm{ac}}(\omega)$

Where $\mathrm{G}_{\mathrm{ac}}(\omega) \approx \omega^{s} 18$

Where $\omega$ is related to the angular frequency of the applied excitation and the exponent " $s$ " is the critical exponent $^{21,24}$

Figure 4. Conductance as a function of frequency of the ITO/ $\beta-\mathrm{CD} / 5 \mathrm{CB} / \beta-\mathrm{CD} / \mathrm{ITO}$ cell a) at the bias voltage $(2 \mathrm{~V})$. b) Conductance as a function of frequency at different applied bias voltages.

It is evident that $G(\omega)$ increases with increasing frequency. Fig. 4(a) shows that the conductance is characterized by the presence of two power laws and a plateau region. This latter corresponds to the frequency independent conductance $\mathrm{G}_{\mathrm{dc}}$ which is related to free charges available in the material. The increase in ac conductance $G_{a c}$ can be attributed to the presence of the charge carriers in the material which can be caused by the reorientation of molecules ${ }^{2,4}$. The first power at low frequency has an exponent close to unity $(0<s<1)^{24}$. The second power, above $10^{5} \mathrm{~Hz}$, with an exponent $s$ in the order of 2, can be related to the series resistance of the ITO electrodes (Fig. 4(a)) ${ }^{21,25}$.
The transition from frequency-independent (dc conductance) to frequency-dependent (ac conductance) behavior indicates the beginning of the relaxation phenomena and is in agreement with the dielectric relaxation behavior studied in the next part. On the other hand, the conductance plot shows a saturated region at high frequencies ${ }^{21,25}$ (Fig. 4(a) lower inset), which indicates that the $\beta$-CD layer has a blocking effect on the electrodes ${ }^{9}$. The conductance variation of the ITO/ $\beta-\mathrm{CD} / \mathrm{LC} / \beta-\mathrm{CD}$ $/$ ITO cell as a function of frequency at different bias voltages is plotted in Fig. 4(b). Fig. 4(b) shows that the conductance decreases with the applied bias voltage. This can be attributed to the migration of 
ionic impurities from the volume to the interfaces. Indeed, the mechanism of generation of charges in the electrodes allows the stabilization of the orientation of the molecules ${ }^{3,19}$.

\section{Admittance measurements}

To better understand the blocking effect, the admittance measurement was carried out. The admittance is proportional to the conductance; it can be written as the following equation ${ }^{25}$.
$\mathrm{Y}(\omega)=\mathrm{G}(\omega)+\mathrm{i} \omega \mathrm{C}$

Where $G(\omega)$ is the equivalent parallel conductance and $C(\omega)$ is the equivalent parallel capacitance related to permittivity and both are in general functions of frequency ${ }^{3}$.

Fig. 5(a) shows that the entire spectra exhibit a single semicircle. The slope of log plot is $\sim 1 / 2$. This is shown in the lower inset of Fig. 5(b).
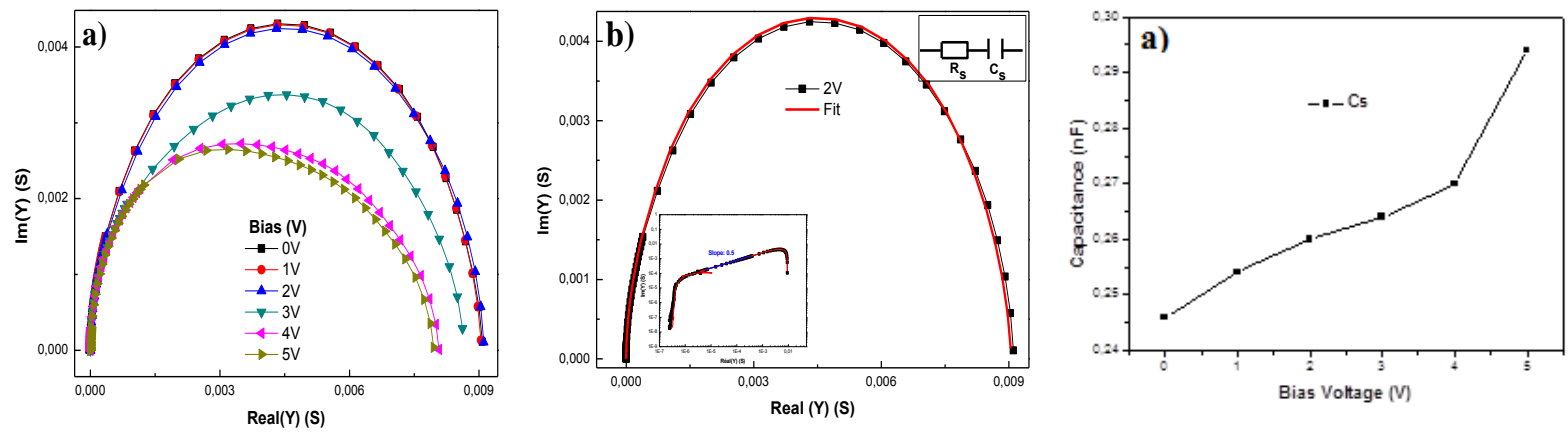

Figure 5. Cole-Cole plots of complex admittance of the ITO $/ \beta-\mathrm{CD} / 5 \mathrm{CB} / \beta-\mathrm{CD} / \mathrm{ITO}$ cell

a) With variation of the bias voltage b) At $2 \mathrm{~V}$, Symbols denote experimental results and line show fit according to the equivalent circuit, the upper inset shows the equivalent circuit and lower inset shows the Cole-Cole plot in logarithmic mode c) Variation of the series capacitance with bias voltage.

The curvature of the semi-circle decreased with increasing the applied dc bias. This decrease is proportional to the decrease of conductance (previous part Fig. 4(b) upper inset). This result can be explained by the blocking effect of alignment layer on the LC cell and the reaction of reorienting molecules which became more stable $21,25,26$. This result is in good agreement with the measurements of conductance. It is clear that the admittance for different values of the applied bias can provide a useful means for the existence of the blockage phenomena ${ }^{25,26}$. The admittance data of the cell at different bias voltages are fitted by the equivalent circuit given in Fig. 5(b) upper inset ${ }^{21}$. The fitting of the experimental data is in good agreement with the theoretical models as shown in Fig. 5(b) [26]. The resulting parameters of the electrical equivalent circuit are summarized in Table 2. The capacitance Cs increases with the increase of the bias voltage (Fig. 5(c)), it amounts to the barrier capacity at high frequency. However, the series resistance Rs is almost independent of the applied bias (Table 2).

Table 2: Fitting parameters of admittance data at different bias voltages.

\begin{tabular}{|lllllll|} 
Bias $(\mathrm{V})$ & 0 & 1 & 2 & 3 & 4 & 5 \\
$\mathrm{R} 1(\Omega)$ & 201.1 & 200.8 & 210.4 & 216.0 & 218.5 & 208.2 \\
\hline $\mathrm{C} 1(\mathrm{nF})$ & 0.246 & 0.254 & 0.260 & 0.264 & 0.270 & 0.294 \\
\hline Chi-Square & $2.9 \mathrm{E}-2$ & $8.48 \mathrm{E}-3$ & $1.86 \mathrm{E}-2$ & $1.63 \mathrm{E}-2$ & $2.41 \mathrm{E}-2$ & $5.32 \mathrm{E}-2$ \\
\hline
\end{tabular}

\section{Impedance spectroscopy}

The measurement of the dielectric relaxation at different frequencies gives information about the dynamics of polar groups and molecular motion ${ }^{3}$. In general, the complex impedance $Z(\omega)$ under a sinusoidal regime can be expressed as the following equation ${ }^{3,26}$ :

$\mathrm{Z}(\omega)=\operatorname{Re}(\mathrm{Z})+j \operatorname{Im}(\mathrm{Z})=\mathrm{Z}^{\prime}+j \mathrm{Z}^{\prime}$

Where $\operatorname{Re}(Z)=Z$ ' and $\operatorname{Im}(Z)=Z$ ', represent real and imaginary parts of the impedance $Z(\omega)$, respectively ${ }^{3}$.
Indeed, the real and imaginary parts of the impedance assigned to the circuit represented in Fig. 6 can be calculated according to the following equations, respectively:

$$
\begin{aligned}
& Z^{\prime}=\mathrm{R}_{\mathrm{s}}+\frac{1}{1+\omega^{2} R_{P 0}^{2} C_{P 0}^{2}}+\frac{1}{1+\omega^{2} R_{P 1}^{2} C_{P 1}^{2}} \\
& -Z^{\prime}=\frac{\omega^{2} R_{P 0}^{2} C_{P 0}^{2}}{1+\omega^{2} R_{P 0}^{2} C_{P 0}^{2}}+\frac{\omega^{2} R_{P 1}^{2} C_{P 1}^{2}}{1+\omega^{2} R_{P 1}^{2} C_{P 1}^{2}}
\end{aligned}
$$

Where $\omega$ is related to the angular frequency of the applied excitation of the circuit. 


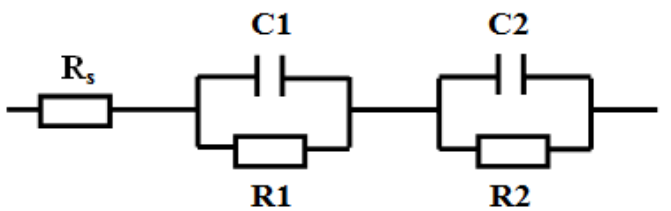

Figure 6. Equivalent electrical circuit model corresponding to the ITO/ $\beta-C D / 5 C B / \beta-C D / I T O$ cell. $R_{s}$ is the resistance of the electrode (ITO). $\mathrm{R}_{1}$ and $\mathrm{C}_{1}$ are associated to the cell volume; $\mathrm{R}_{2}$ and $\mathrm{C}_{2}$ correspond to the $\mathrm{LC} /(\beta$ -

$\mathrm{CD}$ ) interface.

- Variation of the real and imaginary parts of the impedance with frequency

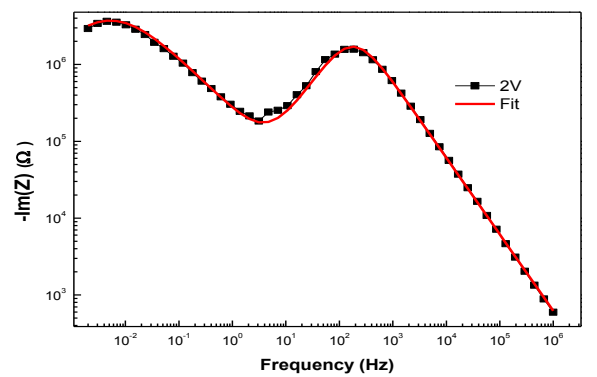

The frequency dependence of the real and imaginary parts of the impedance at $2 \mathrm{~V}$ is shown in Fig. 7. The imaginary impedance part shows two peaks (Fig. 7).

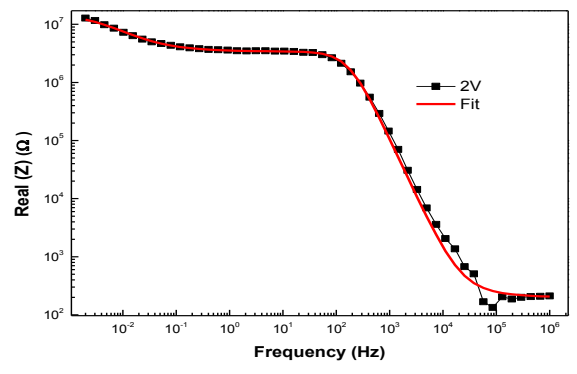

Figure 7. Imaginary and Real parts of the complex impedance as a function of the frequency of the ITO/ $\beta$ $\mathrm{CD} / 5 \mathrm{CB} / \beta-\mathrm{CD} / \mathrm{ITO}$ cell at the bias voltage (2V). Symbols are measured data and the solid lines are the fitting ones.

These peaks prove the existence of two kinds of dielectric relaxation in the sample ${ }^{1,4,9}$. Thus, we can propose that the change in dielectric relaxation is related to the molecular reorientation of LC molecules (dipolar orientation) ${ }^{5,25,27}$ in the highfrequency range which is triggered by external applied voltage ${ }^{1,4,8}$ and an ionic relaxation at the LCelectrodes interfaces in the lower one ${ }^{9,27}$. On the basis of Murakami's study ${ }^{9,28}$, we can consider that the relaxation in the low- frequency domain is due to the formation of Helmholtz double layer at the electrode $/ 5 \mathrm{CB}$ interfaces. The double layer is formed via the adsorption of impurity ions in the LC 5CB onto the electrode surfaces 28 of the cell and therefore the electrode/5CB interface behaves as a parallel-plate capacitor [8,9]. The dielectric relaxation due to the orientational polarizability was

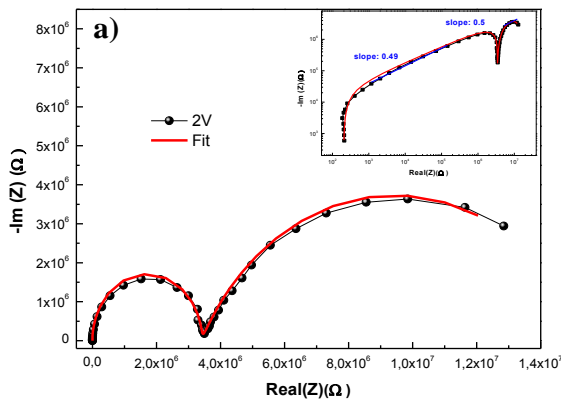

observed in frequency higher than $10^{3} \mathrm{~Hz}$. It provided information on the molecular rotation ${ }^{1,8,28}$. In the low frequency regime $\left(10^{-1}-10^{3} \mathrm{~Hz}\right)$, electrode polarization was observed in NLCs as well as alignment layers $1,19,28$. The occurrence of this relaxation is related to ionic impurities present in the materials ${ }^{2,4}$.

\section{- Complex impedance analysis}

To understand the dynamic response in the liquid crystal cell, a Cole-Cole plot, is shown in Fig. 8(a). Cole-Cole curves give useful information about the relaxation mechanism of the cells ${ }^{3}$. The arc law is due to a distribution of relaxation times ${ }^{3,4,28}$.

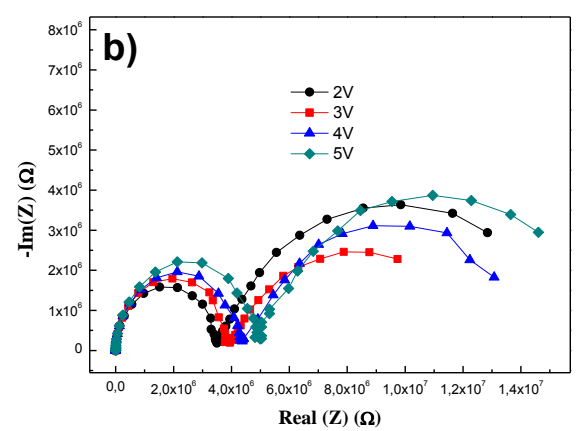

Figure 8. Cole-Cole plots of complex impedance of the ITO $/ \beta-\mathrm{CD} / 5 \mathrm{CB} / \beta-\mathrm{CD} / \mathrm{ITO}$ cell a) at the bias voltage $(2 \mathrm{~V})$. Symbols are measured data and the solid lines are the fitting ones. The upper inset shows the Cole-Cole plot in logarithmic mode with slope $\sim 0.5$ b) at 2, 3, 4 and 5 bias voltages.

The Cole-Cole plot explains the kinds of dielectric relaxation existing in the frequency dependent response of the cell. So, the Cole-Cole plot shows the presence of two dielectric relaxations in the complex impedance at $2 \mathrm{~V}$. As can be seen on 
logarithmic mode, the slope of the plots is $\sim 0.5$ (Fig. 8(a) upper inset) which means that the curvature is semicircle. The first one is a half circle which is supposed to be due to a molecular orientational polarization ${ }^{5,29}$, and the second one is at lowfrequency response (below $100 \mathrm{~Hz}$ ), which is possibly due to an interfacial polarization ${ }^{1,29}$. The dielectric relaxation in the low-frequency regime was attributed to the ionic space-charge polarization or to the electrical double layer formed at the electrode/5CB interface ${ }^{1,9}$. The ionic space-charge polarization arises from long-range movements of impurity ions in 5CB. The Cole-Cole plots have the same behavior for different bias voltages $(2,3,4$ and $5 \mathrm{~V}$ ) (Fig. 8(b)). The impedance values change by a high amount when the applied voltage is increased. The impedance is dependent on the applied voltage. This is due to the anisotropy property of the samples ${ }^{3}$. The plot shows that the first semicircle's curvature (size) increases with increasing bias voltage. This suggests that the relaxation process are associated with the reorientation of LC molecules ${ }^{4,9}$,

however the second semicircle decreases in size with increasing bias voltage, as illustrated in Fig. 8(b).
To gain more information on the relaxation mechanism, we assimilated the LC cell to an electrical equivalent circuit. This latter allows the analysis of the Cole-Cole plot and includes contributions from the electrodes, the LC alignment layer and the LC in the volume of the cell. On account of the existence of two relaxation times, we assign, in the modeling, a parallel resistor capacitor circuit for $L C / \beta-C D$ interface $\left(\mathrm{R}_{2}\right.$ and $\left.\mathrm{C}_{2}\right)$ and another one for the volume $\left(R_{1}\right.$ and $\left.C_{1}\right){ }^{9}$. These circuits are linked serially to a resistance $\mathrm{Rs}$ associated with the ITO electrode, which was found to be approximately $200 \Omega$. The series resistance values Rs are small but they remain constant for higher bias voltages ${ }^{29}$.

\section{- Parameters obtained by modeling the impedance spectra}

The impedance data of the cell at different bias voltages are fitted by the equivalent circuit given in Fig. 6. The fitting of the experimental data is in good agreement with the theoretical models as shown in Fig. 8(a) [6].

Table 3: Fitting parameters of impedance data at different bias voltages.

\begin{tabular}{|lllllll|}
\hline Bias $(\mathrm{V})$ & 0 & 1 & 2 & 3 & 4 & 5 \\
$\mathrm{R}_{\mathrm{s}}(\Omega)$ & 186 & 186.2 & 198.4 & 198.5 & 212.6 & 199.4 \\
\hline $\mathrm{R}_{1}(\mathrm{M} \Omega)$ & 2.845 & 2.893 & 3.42 & 3.81 & 4.23 & 4.85 \\
\hline $\mathrm{C}_{1}(\mathrm{nF})$ & 0.258 & 0.262 & 0.266 & 0.268 & 0.272 & 0.298 \\
\hline $\mathrm{n}_{1}$ & 0.9927 & 1 & 1 & 1 & 1 & 1 \\
\hline $\mathrm{R}_{2}(\mathrm{M} \Omega)$ & $*$ & 73.8 & 12.67 & 9.85 & 9.51 & 8.39 \\
\hline $\mathrm{C}_{2}(\mu \mathrm{F})$ & 0.9673 & 0.9549 & 0.925 & 0.848 & 0.815 & 0.811 \\
\hline $\mathrm{n}_{2}$ & 0.729 & 0.671 & 0.716 & 0.688 & 0.75 & 0.729 \\
\hline Chi-Square & $6.387 \mathrm{E}-2$ & $3.2759 \mathrm{E}-2$ & $6.87 \mathrm{E}-2$ & $5.631 \mathrm{E}-2$ & $1.38 \mathrm{E}-2$ & $1.01 \mathrm{E}-2$ \\
\hline$\tau_{1}(\mathrm{~ms})$ & 0.73401 & 0.75796 & 0.90972 & 1.02108 & 1.15056 & 1.4453 \\
\hline$\tau_{2}(\mathrm{~s})$ & $*$ & 70.471 & 11.719 & 8.3528 & 7.75065 & 6.8042 \\
\hline$f_{1}(\mathrm{~Hz})$ & 216.82 & 210.08 & 175.03 & 155.94 & 138.39 & 110.17 \\
\hline$f_{2}(\mathrm{~Hz})$ & $*$ & 0.00226 & 0.01358 & 0.01906 & 0.020545 & 0.0234025 \\
\hline
\end{tabular}

The resulting parameters of the electrical equivalent circuit are summarized in Table 3 . The capacitance values $\mathrm{C}_{1}$ and $\mathrm{C}_{2}$ are of the order of $\mathrm{nF}$ and $\mu \mathrm{F}$, respectively, which is in agreement with previous works ${ }^{9}$. This is also the case for the high

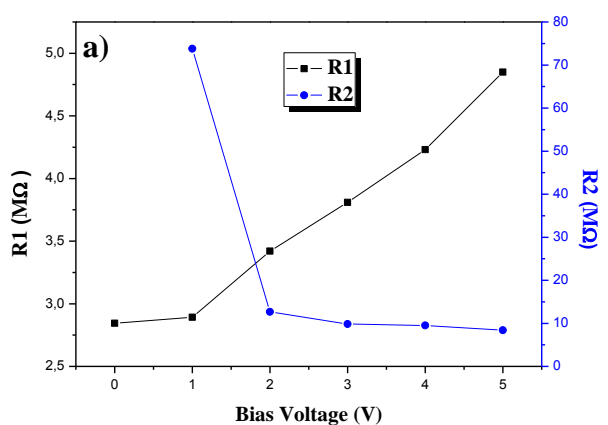

resistance values (order of magnitude of $M \Omega$ ). It is seen that $R_{1}$ values increase with increasing the applied voltage (Fig. 9(a) upper inset). This suggests that the $R_{1}$ values related to the relaxation process are associated with the reorientation of molecules ${ }^{2}$.

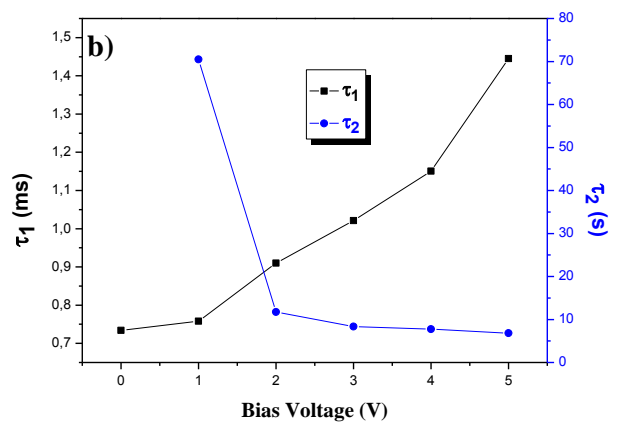

Figure 9. a) Variation of the parallel resistance $\left(\mathrm{R}_{1}, \mathrm{R}_{2}\right)$ with bias voltage $\left.\mathbf{b}\right)$ Variation of the relaxation times $\left(\tau_{1}, \tau_{2}\right)$ with bias voltage. 
Moreover, Fig. 9(a) shows the variation of $R_{2}$ with the applied bias. The resistance $R_{2}$ decreases with the increase of the bias voltage. This is due to the increase in the number of injected carrier into molecules. The carriers may be trapped by structural defects or chemical impurities. The impurity ions in $5 \mathrm{CB}$ adsorb on the electrodes and form Helmholtz double layers. Since some of the ions may be incorporated during the synthesis of $5 \mathrm{CB}$ and cell manufacturing, many ionic species exist in the $5 \mathrm{CB}$ cells.

The Nyquist plot also can give the information about the relaxation time, $\tau$, as in the following equation ${ }^{1,30}$.

$\tau_{\mathrm{i}}=\frac{1}{2 \pi f i}=\frac{1}{\omega i}=\mathrm{RiCi} \quad$ Where $\mathrm{i}=1,2$

The relaxation time $\tau_{1}$ (order of ms) and $\tau_{2}$ (order of s) are suitable for the contribution to the orientationel relaxation and the ionic relaxation, respectively (Table 3$)^{9}$.

The relaxation time $\tau_{1}$ increases with increasing bias voltage (Fig. 9(b)). It suggests that the relaxation process is associated with the rotational motion of the LC molecules. The millisecond values obtained for $\tau_{1}$ indicate that the relaxation is dipolar 1,3 . On the other hand, the relaxation time $\tau_{2}$ decreases with the increase in the bias voltage as given in Fig. 9(b). This behavior can be understood as follows: as the bias voltage is increased, a large number of charge carriers are injected into the devices, resulting in a decrease in the dielectric relaxation time ${ }^{24}$.

For liquid crystal devices, the relaxation time is of extreme importance, which further depends on the cell gap, the degree of molecular alignment and many other parameters. When a high voltage was applied to the sample cell, the relaxation time was affected.

\section{Conclusion}

In this paper, we presented the fabrication and the characteristics of nematic liquid crystal (NLC) cell using Beta Cyclodextrins ( $\beta-\mathrm{CD})$ as alignment layers. We found that the hydrophilic surface and its morphology play an important role in the planar alignment of liquid crystal.

Impedance spectroscopy was used to report the effect of this alignment layer on dielectric properties and the liquid crystal molecular orientation. It was found that this layer has a blocking effect on the LC cell. Therefore, we studied the dielectric relaxation behavior of the cell. Two kinds of relaxation were observed; the first was attributed to the reorientation of the LC molecules in the volume and the second was associated with the ionic relaxation at the LC- $(\beta-$ $\mathrm{CD})$ interfaces of the cell. We obtained good agreement between the experimental and the fitted curves. We also determined the different component parameters of the electrical equivalent circuit and we obtained the expected values.

\section{References}

1- S.S. Parab, M.K. Malik, R.R. Deshmukh, Dielectric relaxation and electro-optical switching behavior of nematic liquid crystal dispersed in poly (methyl methacrylate), J. Non-Cryst. Solids. 358 (2012) 2713-2722.

2- O. Köysal, M. Okutan, M. Gökçen, Investigation of dielectric properties and diffraction efficiency enhancements caused by photothermal effect in DR9 dye-doped nematic liquid crystal, Opt. Commun. 284 (2011) 49244928.

3- M. Okutan, F. Yakuphanoglu, S.E. Sana, O. Koysal, Impedance spectroscopy and dielectric anisotropy-type analysis in dye-doped nematic liquid crystals having different preliminary orientations, Physica B. 368 (2005) 308-317.

4- M. Okutan, F. Yakuphanoglu, O. Koysal, M. Durmus, V. Ahsen, Dielectric spectroscopy analysis in employing liquid crystal phthalonitrile derivative in nematic liquid crystals, Spectrochim. Acta, Part A. 67 (2007) 531-535.

5- F. Al-Hazmi, A. Al-Ghamdi, N. Al-Senany, F. Alnowaiser, F. Yakuphanoglu, Dielectric anisotropy and electrical properties of the copper phthalocyanine (CuPc): 4-40-n Heptylcyanobiphenyl (7CB) composite liquid crystals, Composites Part B. 56 (2014) 15-19.

6- R. Manohar, A.K. Misra, D.P. Singh, S.P. Yadav, P. Tripathi, A.K. Prajapati, M.C.Varia, Dielectric, thermal and optical study of an unusually shaped liquid crystal, J. Phys. Chem. Solids. 71 (2010) 1684-1689.

7- S. Peralta, F. Hapiot, Y. Barbaux, M. Wrentham, Alignment of nematic crystal using substituted calixarene Langmuir-Blodgett films, Liq. Cryst. 30 (2003) 436-469.

8- M. Rahman, C.W. Hsieh, C.T. Wang, B.R. Jian, W. Lee, Dielectric relaxation dynamics in liquid crystal-dye composites, Dyes Pigm. 84 (2010) 128-133.

9- A. Abderrahmen, F. F. Romdhane, H. Ben Ouada, A. Gharbi, Investigation of the liquid crystal alignment layer: effect on electrical properties, Sci. Technol. Adv. Mater. 9 (2008) 025001 .

10- B.J. Shen, Z. Xie, Y.N. He, Y.Q. Lian, Cinnamate-functionalized hyperbranched polymeras liquid crystal photo-alignment layer, Chin. Chem. Lett.19 (2008) 1131-1134.

11- M. Echabaane, A. Rouis, I. Bannamour, H. Ben Ouada, Optical electrical and sensing properties of $\beta$-ketoimine calix[4]arene thin films, Mater. Chem. Phys. 141 (2013) 781-789.

12- A. Gaied, N. Jaballah, S. Teka, M. Majdoub, A water-Insoluble $\beta$-Cyclodextrin Derivative for Hydroquinone Sensor Applications, International Peer Reviewed Journal, 
2014, 3 (4): 1655-1664.

13- R. Mlika, S. Hbaieb, R. Ben Chaabene, Y. Chevalier, R. Kalfat, H. Ben Ouada, Electrochemical properties of gold electrodes functionalized by new pseudo-polyrotaxanes of polyaniline and chemically modified $\beta$-cyclodextrin inclusion complex, Synth. Met. 162 (2012) 186-192.

14- M. Raoov, S. Mohamad, M.R. Abas, Synthesis and characterization of $\beta$-cyclodextrin functionalized ionic liquid polymer as macroporous material for the removal of phenols and As(V), Int. J. Mol. Sci. 15 (2014) 100-119.

15- P. Upadhyay, M.K. Rastogi, D. Kumar, Polarizability study of nematic liquid crystal 4-cyano-40-pentylbiphenyl (5CB) and its nitrogen derivatives, Adv. Chem. Phys. 456 (2015) 41-46.

16- P. Jedlovszky, L.B. Pártay, Investigation of the saturated adsorption layer of 5-cyano-biphenyl and 5-cyano-terphenyl at the free water surface by Monte Carlo simulation, J. Mol. Liq. 136 (2007) 249-256.

17- A. Gunaratne, H. Corke, Effect of hydroxypropyl b-cyclodextrin on physical properties and transition parameters of amylase-lipid complexes of native and acetylated starches, Food Chem. 108 (2008) 14-22.

18- A. Rouis, C. Dridi, I. Bonnamour, J. Davenas, H. Ben Ouada, Transport mechanism and trap distribution in ITO/azo-calix[4]arene derivative/Al diode structure, Physica B. 399 (2007) 109-115.

19- P. K. Tripathi, A. Misra, K. Pandey, R. Manohar, Study on dielectric and optical properties of $\mathrm{ZnO}$ doped nematic liquid crystal in low frequency region, Chem. Commun. 1(2013)20-26.

20- R. Ploeger, S. Musso, O. Chiantore, Contact angle measurements to determine the rate of surface oxidation of artists' alkyd paints during accelerated photo-ageing, Prog. Org. Coat. 65 (2009) 77-83.

21- M.C. Petty, C. Pearson, A.P. Monkman, R. Casalini, S. Capaccioli, J. Nagel,
Application of impedance spectroscopy to the study of organic multilayer devices, Colloids and Surfaces A: Physicochem. Eng. Aspects. 171 (2000) 159-166.

22- R. Mlika, A. Rouis, I. Bonnamour, H. Ben Ouada, Impedance spectroscopic investigation of the effect of thin azo-calix[4]arene film type on the cation sensitivity of the gold electrodes, Mater. Sci. Eng., C. 31 (2011) 1466-1471.

23- Y. Shiraishi, T. Uehara, H. Sawai, H. Kakiuchi, S. Kobayashi, N.Toshima, Electro-optic properties of liquid crystal devices doped withcucurbit(6)uril-protected zirconia nanowires, Colloids and Surfaces A: Physicochem. Eng. Aspects. 460 (2014) 90-94.

24- I. Zahou, R. Ben Chaabane, R. Mlika, S. Touaiti, B. Jamoussi, H. Ben Ouada, Optical and electrical properties of novel peripherally tetra and mono -quinoleinoxy substituted metallophtalocyanines, J Mater Sci: Mater Electron. DOI 10.1007/s10854-014-1955-6.

25- A.K. Jonscher, Alternating Current Diagnostics of Poorly Conducting thin films, Thin Solid Films. 36 (1976) 1-20.

26- R.P. Suvarna, K.R. Rao, K. Subbarangaiah, A simple technique for a.c. conductivity measurements, Bull. Mater. Sci. 25 (2002) 647-651.

27- F.M. Aliev, Z. Nazario, G.P. Sinha, Broadband dielectric spectroscopy of confined liquid crystals, J. Non-Cryst. Solids. 305 (2002) 218225.

28- S. Murakami, H. Iga, H. Naito, Dielectric properties of nematic liquid crystals in the ultralow frequency regime, J. Appl. Phys. 80 (1996) 6396-6400.

29- J. Ahn, D. Chung, J. Lee, G. Lee, M. Song, W. Lee, W. Han, T. Kim, Equivalent-Circuit Analysis of Organic Light-Emitting Diodes by Using the Frequency-Dependent Response of an ITO/Alq3/Al Device, J. Korean. Phys. Soc. 46 (2005) 546-550.

30- M. Kondo, N. Suzuki, Y. Nakajima, T. Tanaka, T. Muroga, Electrochemical impedance spectroscopy on in-situ analysis of oxide layer formation in liquid metal, Fusion Eng. Des. 89(2014)1201-1208. 